EP-84

\title{
Microsurgical hepatic artery reconstruction in deceased donor liver transplantation for reduced arterial complications
}

\author{
Lee SUNG-GYU*, Young-In YOON
}

Division of Hepatobiliary Surgery and Liver Transplantation, Asan Medical Center, University of Ulsan College of Medicine, Seoul, Korea

Introduction: Aberrant donor hepatic artery anatomy or hepatic artery injury during organ procurement or recipient preparation poses a surgical challenge during deceased donor liver transplantation. In this study, we aimed to investigate arterial reconstruction using microvascular techniques during deceased donor liver transplantation and to suggest reasonable indications for the microsurgical approach in this setting.

Methods: We retrospectively reviewed the outcomes of 470 deceased donor liver transplantations performed at our institution between July 2011 and December 2015. Of these, 128 recipients underwent microsurgical hepatic artery reconstruction and 342 underwent reconstruction with surgical loupes.

Results: Thirty-two patients (6.8\%) experienced hepatic artery-related complications, including hepatic artery thrombosis $(\mathrm{n}=8,1.7 \%)$. In the propensity score-matched cohort, the surgical loupe group showed a higher complication rate $(p=0.782)$. On multivariate analysis, cold ischemia time (odds ratio, 0.995; 95\% confidence interval, 0.992-0.999; $p=0.009$ ) and use of aortohepatic conduits (odds ratio, 5.254; 95\% confidence interval, $1.878-14.699 ; p=0.002$ ) were independent predictors of arterial complications.

Conclusions: The low incidence of hepatic artery complications in this study is likely attributable to the active application of microsurgical techniques. Active application of backtable microsurgical plasty and selective application of microsurgical techniques for main arterial reconstruction may help minimize operative difficulties and arterial complications. 